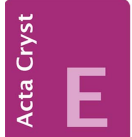

CRYSTALLOGRAPHIC COMMUNICATIONS

ISSN 2056-9890

\section{Crystal structure of cyproconazole}

\author{
Gihaeng Kang, Jineun Kim,* Eunjin Kwon and Tae Ho \\ Kim*
}

Department of Chemistry and Research Institute of Natural Sciences, Gyeongsang National University, Jinju 52828, Republic of Korea. *Correspondence e-mail:

thkim@gnu.ac.kr, jekim@gnu.ac.kr

Received 16 November 2015; accepted 26 November 2015

Edited by P. C. Healy, Griffith University, Australia

The title compound [systematic name: 2-(4-chlorophenyl)3-cyclopropyl-1-(1H-1,2,4-triazol-1-yl)butan-2-ol], $\quad \mathrm{C}_{15} \mathrm{H}_{18} \mathrm{Cl}$ $\mathrm{N}_{3} \mathrm{O}$, is a conazole fungicide. The asymmetric unit comprises two enantiomeric pairs (molecules $A$ and $B$ ) in which the dihedral angles between the chlorophenyl and triazole rings are 46.54 (9) (molecule $A$ ) and $67.03(8)^{\circ}$ (molecule $B$ ). In the crystal, $\mathrm{C}-\mathrm{H} \cdots \mathrm{O}, \mathrm{O}-\mathrm{H} \cdots \mathrm{N}$ and $\mathrm{C}-\mathrm{H} \cdots \mathrm{Cl}$ hydrogen bonds and weak $\mathrm{C}-\mathrm{H} \cdots \pi$ interactions [3.473 (2) $\AA$ ] link adjacent molecules, forming columns along the $a$ axis.

Keywords: crystal structure; cyproconazole; butan-2-ol; fungicidal properties; hydrogen bonding.

CCDC reference: 1439054

\section{Related literature}

For information on the fungicidal properties of the title compound, see: Hester et al. (2012). For a related crystal structure, see: Chopra et al. (2004).

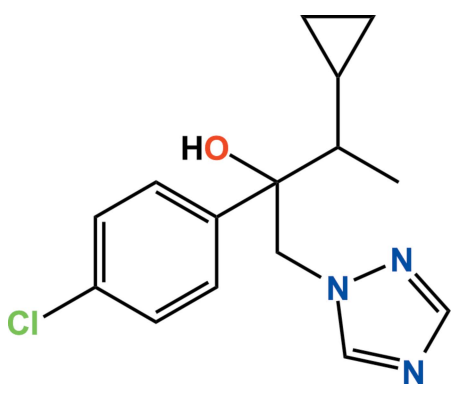

\section{Experimental}

\subsection{Crystal data}

$\mathrm{C}_{15} \mathrm{H}_{18} \mathrm{ClN}_{3} \mathrm{O}$

$M_{r}=291.77$

Triclinic, $P \overline{1}$

$a=9.7783$ (11) ̊ $b=12.2150(13) \AA$

$c=14.5861(15) \AA$

$\alpha=107.965(6)^{\circ}$

$\beta=108.254(5)^{\circ}$

$\gamma=99.533(5)^{\circ}$

$V=1506.1(3) \AA^{3}$

$Z=4$

Mo $K \alpha$ radiation

$\mu=0.25 \mathrm{~mm}^{-1}$

$T=173 \mathrm{~K}$

$0.27 \times 0.24 \times 0.13 \mathrm{~mm}$

\subsection{Data collection}

Bruker APEXII CCD diffractometer

Absorption correction: multi-scan

(SADABS; Bruker, 2013)

$T_{\min }=0.657, T_{\max }=0.746$

\subsection{Refinement}

$R\left[F^{2}>2 \sigma\left(F^{2}\right)\right]=0.048$

$w R\left(F^{2}\right)=0.142$

$S=1.10$

5902 reflections
365 parameters

$\mathrm{H}$-atom parameters constrained

$\Delta \rho_{\max }=0.37 \mathrm{e}^{-3}$

$\Delta \rho_{\min }=-0.42 \mathrm{e}^{-3}$
Table 1

Hydrogen-bond geometry $\left(\AA{ }^{\circ}\right)$.

$C g 1$ is the centroid of the $\mathrm{N} 1 / \mathrm{N} 2 / \mathrm{C} 15 / \mathrm{N} 3 / \mathrm{C} 14$ ring.

\begin{tabular}{|c|c|c|c|c|}
\hline$D-\mathrm{H} \cdots A$ & $D-\mathrm{H}$ & $\mathrm{H} \cdots A$ & $D \cdots A$ & $D-\mathrm{H} \cdots A$ \\
\hline $\mathrm{O} 1-\mathrm{H} 1 \cdots \mathrm{N} 6^{\mathrm{i}}$ & 0.84 & 2.05 & $2.884(2)$ & 171 \\
\hline $\mathrm{O} 2-\mathrm{H} 2 \cdots \mathrm{N} 3^{\mathrm{ii}}$ & 0.84 & 2.07 & $2.856(2)$ & 156 \\
\hline $\mathrm{C} 8-\mathrm{H} 8 \cdots \mathrm{Cl} 1^{\mathrm{iii}}$ & 1.00 & 2.83 & $3.633(2)$ & 137 \\
\hline $\mathrm{C} 15-\mathrm{H} 15 \cdots \mathrm{O} 2^{\mathrm{iv}}$ & 0.95 & 2.53 & $3.284(2)$ & 137 \\
\hline $\mathrm{C} 13-\mathrm{H} 13 B \cdots C g 1^{\mathrm{v}}$ & 1.00 & 2.91 & $3.473(2)$ & 117 \\
\hline
\end{tabular}

Data collection: APEX2 (Bruker, 2013); cell refinement: SAINT (Bruker, 2013); data reduction: $S A I N T$; $\operatorname{program}(\mathrm{s})$ used to solve structure: SHELXS97 (Sheldrick, 2008); program(s) used to refine structure: SHELXL2013 (Sheldrick, 2015); molecular graphics: DIAMOND (Brandenburg, 2010); software used to prepare material for publication: SHELXTL (Sheldrick, 2008).

\section{Acknowledgements}

This research was supported by the Basic Science Research Program through the National Research Foundation of Korea (NRF) funded by the Ministry of Education, Science and Technology (No. 2015R1D1A4A01020317).

Supporting information for this paper is available from the IUCr electronic archives (Reference: HG5465).

\section{References}

Brandenburg, K. (2010). DIAMOND. Crystal Impact GbR, Bonn, Germany. Bruker (2013). APEX2, SAINT and SADABS. Bruker AXS Inc., Madison, Wisconsin, USA.

Chopra, D., Mohan, T. P., Rao, K. S. \& Guru Row, T. N. (2004). Acta Cryst. E60, o2410-o2412.

Hester, S., Moore, T., Padgett, W. T., Murphy, L., Wood, C. E. \& Nesnow, S. (2012). Toxicol. Sci. 127, 54-65.

Sheldrick, G. M. (2008). Acta Cryst. A64, 112-122.

Sheldrick, G. M. (2015). Acta Cryst. C71, 3-8. 


\section{supporting information}

Acta Cryst. (2015). E71, o1007 [https://doi.org/10.1107/S2056989015022665]

\section{Crystal structure of cyproconazole}

\section{Gihaeng Kang, Jineun Kim, Eunjin Kwon and Tae Ho Kim}

\section{S1. Comment}

Cyproconazole [systematic name: 2-(4-chlorophenyl)-3-cyclopropyl-1-(1H-1,2,4-triazol-1-yl)butan-2-ol] is a conazole fungicide used as agricultural pesticides and pharmaceutical products. (Hester et al., 2012). Its crystal structure is reported herein. In this compound (Fig. 1), there are two enantiomeric pairs (Molecule A and B) in the asymmetric unit, with the dihedral angles between the chlorophenyl and triazole rings are 46.54 (9) (Molecule A) and 67.03 (8) (Molecule B), respectively. All bond lengths and bond angles are normal and comparable to those observed in the crystal structure of a similar compound (Chopra et al., 2004).

In the crystal structure (Fig. 2), the crystal structure is stabilized by $\mathrm{C}-\mathrm{H} \cdots \mathrm{O}, \mathrm{O}-\mathrm{H} \cdots \mathrm{N}$, and $\mathrm{C}-\mathrm{H} \cdots \mathrm{Cl}$ hydrogen bonds (Table 1), as well as intermolecular $\mathrm{C} 13-\mathrm{H} 13 \mathrm{~B} \cdots C g 1^{\mathrm{v}}(\mathrm{Cg} 1$ is the centroid of the $\mathrm{N} 1-\mathrm{N} 2-\mathrm{C} 15-\mathrm{N} 3-\mathrm{C} 14$ ring) interaction with a triazole ring are present, resulting in one-dimensional columns along to $a$-axis [for symmetry code: (v), $-x+1,-y+1,-z]$.

\section{S2. Experimental}

The title compound was purchased from the Dr. Ehrenstorfer $\mathrm{GmbH}$ Company. Slow evaporation of a solution in $\mathrm{CH}_{2} \mathrm{Cl}_{2}$ gave single crystals suitable for X-ray analysis.

\section{S3. Refinement}

All H-atoms were positioned geometrically and refined using a riding model with $\mathrm{d}(\mathrm{O}-\mathrm{H})=0.84 \AA, U_{\text {iso }}=1.5 U_{\text {eq }}(\mathrm{C})$ for $\mathrm{O}-\mathrm{H}$ group, $\mathrm{d}(\mathrm{C}-\mathrm{H})=1.00 \AA, U_{\text {iso }}=1.2 U_{\mathrm{eq}}(\mathrm{C})$ for $\mathrm{Csp} p^{3}-\mathrm{H}$ group, $\mathrm{d}(\mathrm{C}-\mathrm{H})=0.99 \AA, U_{\text {iso }}=1.2 U_{\text {eq }}(\mathrm{C})$ for $\mathrm{CH}_{2}$ group, $\mathrm{d}(\mathrm{C}-\mathrm{H})=0.98 \AA, U_{\text {iso }}=1.5 U_{\text {eq }}(\mathrm{C})$ for $\mathrm{CH}_{3}$ group, $\mathrm{d}(\mathrm{C}-\mathrm{H})=0.95 \AA, U_{\text {iso }}=1.2 U_{\text {eq }}(\mathrm{C})$ for aromatic $\mathrm{C}-\mathrm{H}$. 


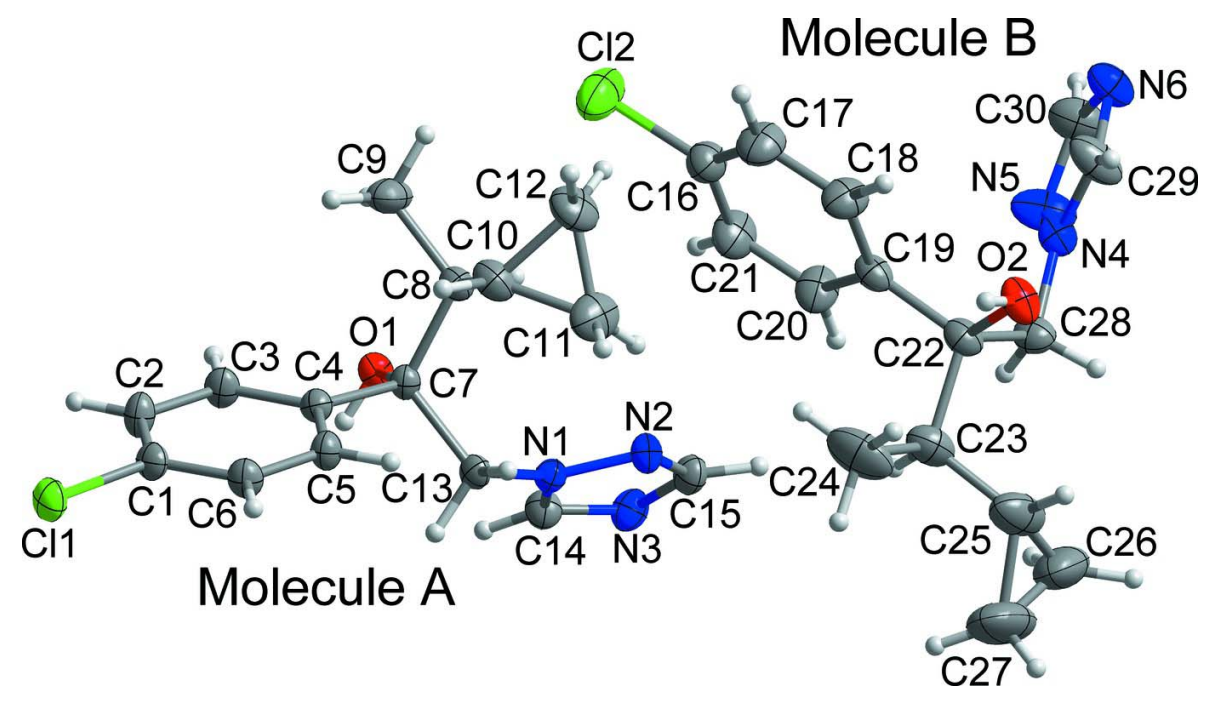

Figure 1

The asymmetric unit of the title compound with the atom-numbering scheme. Displacement ellipsoids are drawn at the $50 \%$ probability level. $\mathrm{H}$ atoms are shown as small spheres of arbitrary radius.

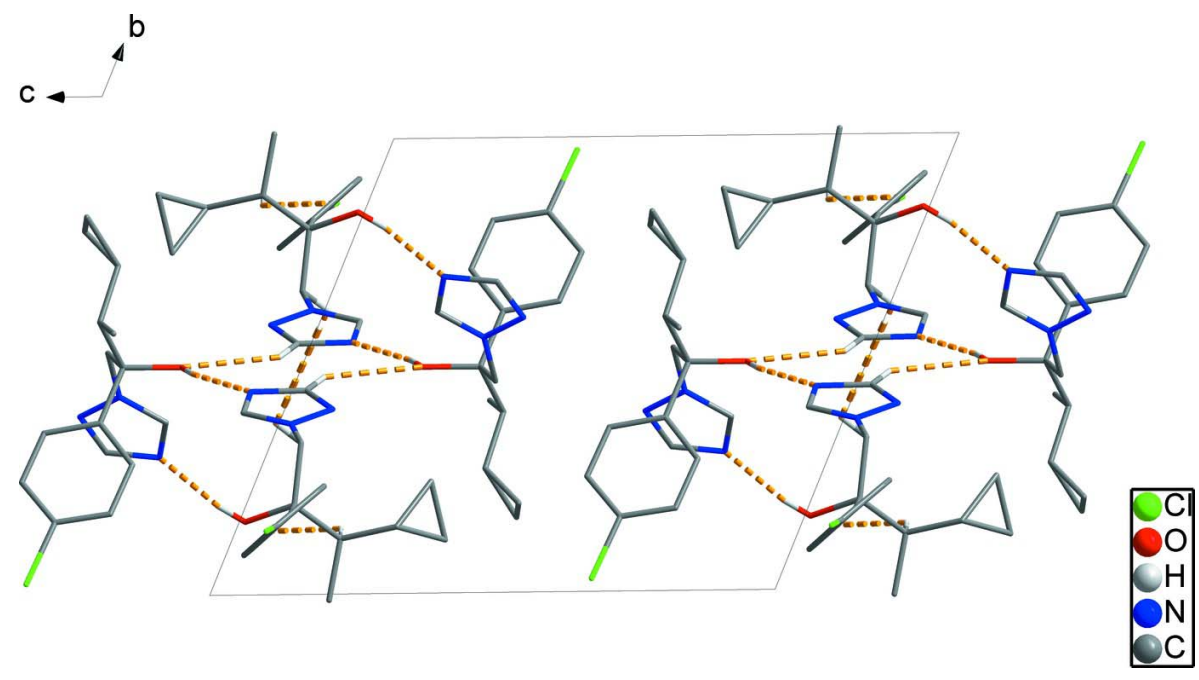

Figure 2

Crystal packing viewed along the $a$ axis. The intermolecular interactions are shown as dashed lines.

2-(4-Chlorophenyl)-3-cyclopropyl-1-(1H-1,2,4-triazol-1-yl)butan-2-ol

Crystal data

$\mathrm{C}_{15} \mathrm{H}_{18} \mathrm{ClN}_{3} \mathrm{O}$

$M_{r}=291.77$

Triclinic, $P \overline{1}$

$a=9.7783(11) \AA$

$b=12.2150(13) \AA$

$c=14.5861(15) \AA$

$\alpha=107.965(6)^{\circ}$

$\beta=108.254(5)^{\circ}$

$\gamma=99.533(5)^{\circ}$

$V=1506.1(3) \AA^{3}$
$Z=4$

$F(000)=616$

$D_{\mathrm{x}}=1.287 \mathrm{Mg} \mathrm{m}^{-3}$

Mo $K \alpha$ radiation, $\lambda=0.71073 \AA$

Cell parameters from 6577 reflections

$\theta=2.2-27.7^{\circ}$

$\mu=0.25 \mathrm{~mm}^{-1}$

$T=173 \mathrm{~K}$

Block, colourless

$0.27 \times 0.24 \times 0.13 \mathrm{~mm}$ 


\section{Data collection \\ Bruker APEXII CCD diffractometer \\ $\varphi$ and $\omega$ scans \\ Absorption correction: multi-scan \\ (SADABS; Bruker, 2013) \\ $T_{\min }=0.657, T_{\max }=0.746$ \\ 24278 measured reflections}

\section{Refinement}

Refinement on $F^{2}$

Least-squares matrix: full

$R\left[F^{2}>2 \sigma\left(F^{2}\right)\right]=0.048$

$w R\left(F^{2}\right)=0.142$

$S=1.10$

5902 reflections

365 parameters

0 restraints
5902 independent reflections

4637 reflections with $I>2 \sigma(I)$

$R_{\text {int }}=0.047$

$\theta_{\text {max }}=26.0^{\circ}, \theta_{\min }=1.6^{\circ}$

$h=-12 \rightarrow 12$

$k=-15 \rightarrow 15$

$l=-17 \rightarrow 17$

Hydrogen site location: inferred from neighbouring sites

$\mathrm{H}$-atom parameters constrained

$w=1 /\left[\sigma^{2}\left(F_{\mathrm{o}}^{2}\right)+(0.0829 P)^{2}\right]$

where $P=\left(F_{\mathrm{o}}{ }^{2}+2 F_{\mathrm{c}}{ }^{2}\right) / 3$

$(\Delta / \sigma)_{\max }<0.001$

$\Delta \rho_{\max }=0.37 \mathrm{e} \AA^{-3}$

$\Delta \rho_{\min }=-0.42$ e $\AA^{-3}$

Special details

Geometry. All e.s.d.'s (except the e.s.d. in the dihedral angle between two 1.s. planes) are estimated using the full covariance matrix. The cell e.s.d.'s are taken into account individually in the estimation of e.s.d.'s in distances, angles and torsion angles; correlations between e.s.d.'s in cell parameters are only used when they are defined by crystal symmetry. An approximate (isotropic) treatment of cell e.s.d.'s is used for estimating e.s.d.'s involving 1.s. planes.

Fractional atomic coordinates and isotropic or equivalent isotropic displacement parameters $\left(\AA^{2}\right)$

\begin{tabular}{lllll}
\hline & $x$ & $y$ & $z$ & $U_{\mathrm{iso}}^{*} / U_{\mathrm{eq}}$ \\
\hline $\mathrm{C} 11$ & $-0.04911(5)$ & $0.86025(4)$ & $0.05469(4)$ & $0.03034(16)$ \\
$\mathrm{C} 2$ & $0.80245(8)$ & $0.97120(5)$ & $0.66620(5)$ & $0.0565(2)$ \\
$\mathrm{O} 1$ & $0.60192(13)$ & $0.83935(11)$ & $0.01077(9)$ & $0.0229(3)$ \\
$\mathrm{H} 1$ & 0.5303 & 0.8046 & -0.0480 & $0.034^{*}$ \\
$\mathrm{O} 2$ & $0.92927(15)$ & $0.49775(13)$ & $0.78653(10)$ & $0.0331(3)$ \\
$\mathrm{H} 2$ & 0.8646 & 0.5125 & 0.8114 & $0.050^{*}$ \\
$\mathrm{~N} 1$ & $0.65641(16)$ & $0.62487(13)$ & $0.02174(12)$ & $0.0224(3)$ \\
$\mathrm{N} 2$ & $0.76461(17)$ & $0.59337(14)$ & $0.08493(12)$ & $0.0256(4)$ \\
$\mathrm{N} 3$ & $0.77957(19)$ & $0.55315(14)$ & $-0.07288(13)$ & $0.0301(4)$ \\
N4 & $1.14669(17)$ & $0.56158(14)$ & $0.69780(12)$ & $0.0278(4)$ \\
N5 & $1.2121(2)$ & $0.60435(18)$ & $0.64189(14)$ & $0.0476(6)$ \\
N6 & $1.35778(19)$ & $0.69573(17)$ & $0.81207(13)$ & $0.0380(4)$ \\
C1 & $0.1300(2)$ & $0.84834(16)$ & $0.06922(14)$ & $0.0243(4)$ \\
C2 & $0.2145(2)$ & $0.91250(17)$ & $0.03227(15)$ & $0.0281(4)$ \\
H2A & 0.1766 & 0.9649 & 0.0014 & $0.034^{*}$ \\
C3 & $0.3549(2)$ & $0.89902(17)$ & $0.04108(15)$ & $0.0273(4)$ \\
H3 & 0.4130 & 0.9431 & 0.0157 & $0.033^{*}$ \\
C4 & $0.41388(19)$ & $0.82362(16)$ & $0.08560(14)$ & $0.0208(4)$ \\
C5 & $0.3266(2)$ & $0.76251(16)$ & $0.12487(14)$ & $0.0244(4)$ \\
H5 & 0.3652 & 0.7117 & 0.1576 & $0.029^{*}$ \\
C6 & $0.1865(2)$ & $0.77470(17)$ & $0.11700(15)$ & $0.0259(4)$ \\
H6 & 0.1290 & 0.7329 & 0.1442 & $0.031^{*}$ \\
& & & &
\end{tabular}




\begin{tabular}{|c|c|c|c|c|}
\hline C7 & $0.56362(19)$ & $0.80332(16)$ & $0.08570(13)$ & 0.0198 \\
\hline C8 & $0.6976(2)$ & $0.87845(16)$ & $0.19188(14)$ & $0.0233(4)$ \\
\hline $\mathrm{H} 8$ & 0.7890 & 0.8564 & 0.1850 & $0.028^{*}$ \\
\hline C9 & $0.7281(3)$ & $1.01291(18)$ & $0.21553(17)$ & $0.0390(5)$ \\
\hline H9A & 0.6417 & 1.0384 & 0.2248 & $0.059 *$ \\
\hline H9B & 0.7438 & 1.0291 & 0.1573 & $0.059^{*}$ \\
\hline $\mathrm{H} 9 \mathrm{C}$ & 0.8183 & 1.0575 & 0.2797 & $0.059^{*}$ \\
\hline C10 & $0.6798(2)$ & $0.85291(18)$ & $0.28317(15)$ & $0.0311(5)$ \\
\hline H10 & 0.5898 & 0.8689 & 0.2972 & $0.037 *$ \\
\hline C11 & $0.7297(3)$ & $0.7547(2)$ & $0.31196(17)$ & $0.0458(6)$ \\
\hline H11A & 0.6697 & 0.7110 & 0.3401 & $0.055^{*}$ \\
\hline H11B & 0.7740 & 0.7052 & 0.2683 & $0.055^{*}$ \\
\hline $\mathrm{C} 12$ & $0.8179(3)$ & $0.8827(2)$ & $0.37830(17)$ & $0.0440(6)$ \\
\hline $\mathrm{H} 12 \mathrm{~A}$ & 0.9166 & 0.9123 & 0.3757 & $0.053^{*}$ \\
\hline H12B & 0.8123 & 0.9182 & 0.4474 & $0.053^{*}$ \\
\hline $\mathrm{C} 13$ & $0.5409(2)$ & $0.66645(16)$ & $0.05308(14)$ & $0.0221(4)$ \\
\hline H13A & 0.5377 & 0.6444 & 0.1124 & $0.027^{*}$ \\
\hline H13B & 0.4421 & 0.6236 & -0.0058 & $0.027^{*}$ \\
\hline $\mathrm{C} 14$ & $0.6682(2)$ & $0.59944(17)$ & $-0.07065(15)$ & $0.0283(4)$ \\
\hline H14 & 0.6042 & 0.6131 & -0.1276 & $0.034 *$ \\
\hline $\mathrm{C} 15$ & $0.8348(2)$ & $0.55172(16)$ & $0.02459(15)$ & $0.0272(4)$ \\
\hline H15 & 0.9183 & 0.5226 & 0.0474 & $0.033^{*}$ \\
\hline $\mathrm{C} 16$ & $0.8286(2)$ & $0.83582(19)$ & $0.67320(16)$ & $0.0361(5)$ \\
\hline C17 & $0.8550(3)$ & $0.8183(2)$ & $0.76483(17)$ & $0.0423(6)$ \\
\hline H17 & 0.8588 & 0.8798 & 0.8251 & $0.051 *$ \\
\hline $\mathrm{C} 18$ & $0.8759(2)$ & $0.71082(19)$ & $0.76897(15)$ & $0.0364(5)$ \\
\hline H18 & 0.8973 & 0.7001 & 0.8333 & $0.044 *$ \\
\hline C19 & 0.8663 (2) & 0.61851 (17) & $0.68184(14)$ & 0.0267 (4) \\
\hline $\mathrm{C} 20$ & $0.8401(3)$ & $0.6393(2)$ & $0.58996(16)$ & $0.0372(5)$ \\
\hline $\mathrm{H} 20$ & 0.8347 & 0.5777 & 0.5292 & $0.045^{*}$ \\
\hline $\mathrm{C} 21$ & $0.8219(3)$ & $0.7468(2)$ & $0.58518(16)$ & $0.0412(6)$ \\
\hline $\mathrm{H} 21$ & 0.8048 & 0.7596 & 0.5220 & $0.049 *$ \\
\hline $\mathrm{C} 22$ & $0.8813(2)$ & $0.49691(18)$ & $0.68326(14)$ & $0.0283(5)$ \\
\hline $\mathrm{C} 23$ & $0.7293(2)$ & $0.3970(2)$ & $0.60963(17)$ & $0.0390(5)$ \\
\hline $\mathrm{H} 23$ & 0.7046 & 0.3983 & 0.5381 & $0.047 *$ \\
\hline $\mathrm{C} 24$ & $0.6022(3)$ & $0.4221(3)$ & $0.6419(2)$ & $0.0642(8)$ \\
\hline $\mathrm{H} 24 \mathrm{~A}$ & 0.5083 & 0.3594 & 0.5926 & $0.096^{*}$ \\
\hline $\mathrm{H} 24 \mathrm{~B}$ & 0.5923 & 0.5006 & 0.6418 & $0.096^{*}$ \\
\hline $\mathrm{H} 24 \mathrm{C}$ & 0.6232 & 0.4226 & 0.7123 & $0.096^{*}$ \\
\hline $\mathrm{C} 25$ & 0.7417 (3) & $0.2731(2)$ & $0.6014(2)$ & $0.0571(8)$ \\
\hline $\mathrm{H} 25$ & 0.7784 & 0.2627 & 0.6695 & $0.069 *$ \\
\hline $\mathrm{C} 26$ & $0.7812(4)$ & $0.1962(2)$ & $0.5174(2)$ & $0.0732(10)$ \\
\hline $\mathrm{H} 26 \mathrm{~A}$ & 0.8009 & 0.2299 & 0.4676 & $0.088^{*}$ \\
\hline H26B & 0.8432 & 0.1436 & 0.5351 & $0.088^{*}$ \\
\hline C27 & $0.6240(4)$ & $0.1651(3)$ & $0.5110(3)$ & $0.0892(12)$ \\
\hline $\mathrm{H} 27 \mathrm{~A}$ & 0.5887 & 0.0936 & 0.5250 & $0.107^{*}$ \\
\hline H27B & 0.5463 & 0.1799 & 0.4574 & $0.107^{*}$ \\
\hline $\mathrm{C} 28$ & $1.0042(2)$ & $0.46528(18)$ & 0.64609 (15) & $0.0312(5)$ \\
\hline
\end{tabular}




\begin{tabular}{lllll}
$\mathrm{H} 28 \mathrm{~A}$ & 1.0226 & 0.3928 & 0.6584 & $0.037^{*}$ \\
$\mathrm{H} 28 \mathrm{~B}$ & 0.9680 & 0.4451 & 0.5698 & $0.037^{*}$ \\
$\mathrm{C} 29$ & $1.2336(2)$ & $0.6183(2)$ & $0.79826(17)$ & $0.0452(6)$ \\
$\mathrm{H} 29$ & 1.2103 & 0.6053 & 0.8533 & $0.054^{*}$ \\
$\mathrm{C} 30$ & $1.3371(3)$ & $0.6832(2)$ & $0.71451(17)$ & $0.0427(6)$ \\
$\mathrm{H} 30$ & 1.4084 & 0.7287 & 0.6985 & $0.051^{*}$ \\
\hline
\end{tabular}

Atomic displacement parameters $\left(\AA^{2}\right)$

\begin{tabular}{|c|c|c|c|c|c|c|}
\hline & $U^{11}$ & $U^{22}$ & $U^{33}$ & $U^{12}$ & $U^{13}$ & $U^{23}$ \\
\hline $\mathrm{C} 11$ & 0.0238 & 0.0358 & $0.0387(3)$ & $0.0144(2)$ & $0.0170(2)$ & $0.0158(2)$ \\
\hline $\mathrm{Cl} 2$ & $0.0824(5)$ & $0.0425(4)$ & $0.0454(4)$ & $0.0272(3)$ & 0.0187 (3) & $0.0196(3)$ \\
\hline $\mathrm{O} 1$ & $0.0216(7)$ & $0.0292(7)$ & $0.0215(6)$ & $0.0068(5)$ & $0.0103(5)$ & $0.0126(5)$ \\
\hline $\mathrm{O} 2$ & $0.0312(8)$ & $0.0497(9)$ & $0.0256(7)$ & $0.0156(7)$ & $0.0137(6)$ & $0.0189(6)$ \\
\hline N1 & $0.0230(8)$ & $0.0218(8)$ & $0.0242(8)$ & $0.0085(6)$ & $0.0101(7)$ & $0.0091(6)$ \\
\hline N2 & $0.0243(8)$ & $0.0296(9)$ & $0.0269(9)$ & $0.0123(7)$ & $0.0103(7)$ & $0.0133(7)$ \\
\hline N3 & $0.0362(10)$ & $0.0304(9)$ & $0.0309(9)$ & $0.0152(7)$ & $0.0184(8)$ & $0.0127(7)$ \\
\hline N4 & $0.0270(9)$ & $0.0344(9)$ & $0.0236(8)$ & $0.0104(7)$ & $0.0116(7)$ & $0.0108(7)$ \\
\hline N5 & $0.0502(12)$ & $0.0563(13)$ & $0.0248(9)$ & $-0.0060(10)$ & $0.0203(9)$ & $0.0062(8)$ \\
\hline N6 & $0.0299(10)$ & $0.0504(11)$ & $0.0298(10)$ & $0.0053(8)$ & $0.0076(8)$ & $0.0180(8)$ \\
\hline $\mathrm{C} 1$ & $0.0211(9)$ & $0.0265(10)$ & $0.0283(10)$ & $0.0104(7)$ & $0.0132(8)$ & $0.0089(8)$ \\
\hline $\mathrm{C} 2$ & 0.0303 (11) & $0.0330(11)$ & $0.0340(11)$ & $0.0166(9)$ & $0.0169(9)$ & $0.0211(9)$ \\
\hline $\mathrm{C} 3$ & $0.0250(10)$ & $0.0298(10)$ & $0.0356(11)$ & $0.0096(8)$ & $0.0165(9)$ & $0.0180(9)$ \\
\hline $\mathrm{C} 4$ & $0.0206(9)$ & $0.0215(9)$ & $0.0206(9)$ & $0.0061(7)$ & $0.0091(7)$ & $0.0071(7)$ \\
\hline $\mathrm{C} 5$ & $0.0256(10)$ & $0.0270(10)$ & $0.0266(10)$ & $0.0113(8)$ & $0.0122(8)$ & $0.0142(8)$ \\
\hline C6 & $0.0244(10)$ & $0.0303(10)$ & $0.0294(10)$ & $0.0086(8)$ & $0.0143(8)$ & $0.0153(8)$ \\
\hline $\mathrm{C} 7$ & $0.0194(9)$ & $0.0227(9)$ & $0.0222(9)$ & $0.0078(7)$ & $0.0104(7)$ & $0.0119(7)$ \\
\hline $\mathrm{C} 8$ & $0.0222(9)$ & $0.0261(10)$ & 0.0207 (9) & $0.0066(7)$ & $0.0080(8)$ & $0.0085(7)$ \\
\hline C9 & $0.0430(13)$ & $0.0281(11)$ & $0.0307(11)$ & $0.0020(9)$ & $0.0041(10)$ & $0.0062(9)$ \\
\hline $\mathrm{C} 10$ & $0.0278(10)$ & $0.0427(12)$ & $0.0238(10)$ & $0.0084(9)$ & $0.0115(9)$ & $0.0134(9)$ \\
\hline C11 & 0.0645 (16) & $0.0458(14)$ & $0.0286(11)$ & $0.0150(12)$ & 0.0147 (11) & $0.0201(10)$ \\
\hline $\mathrm{C} 12$ & $0.0447(14)$ & $0.0547(15)$ & $0.0246(11)$ & $0.0090(11)$ & $0.0057(10)$ & $0.0158(10)$ \\
\hline $\mathrm{C} 13$ & $0.0198(9)$ & $0.0236(9)$ & $0.0253(9)$ & $0.0078(7)$ & $0.0110(8)$ & $0.0093(8)$ \\
\hline $\mathrm{C} 14$ & $0.0310(11)$ & $0.0308(11)$ & $0.0244(10)$ & $0.0118(8)$ & $0.0112(9)$ & $0.0107(8)$ \\
\hline $\mathrm{C} 15$ & $0.0264(10)$ & $0.0271(10)$ & $0.0331(11)$ & $0.0119(8)$ & $0.0147(9)$ & $0.0126(8)$ \\
\hline $\mathrm{C} 16$ & $0.0406(12)$ & $0.0354(12)$ & $0.0302(11)$ & $0.0120(9)$ & $0.0109(10)$ & $0.0124(9)$ \\
\hline $\mathrm{C} 17$ & $0.0578(15)$ & $0.0382(13)$ & $0.0262(11)$ & 0.0153 (11) & $0.0161(11)$ & $0.0059(9)$ \\
\hline $\mathrm{C} 18$ & $0.0492(13)$ & $0.0363(12)$ & $0.0218(10)$ & $0.0097(10)$ & $0.0135(10)$ & $0.0104(9)$ \\
\hline C19 & $0.0240(10)$ & $0.0354(11)$ & $0.0182(9)$ & $0.0078(8)$ & $0.0078(8)$ & $0.0079(8)$ \\
\hline $\mathrm{C} 20$ & $0.0515(14)$ & 0.0429 (13) & $0.0230(10)$ & $0.0207(10)$ & $0.0185(10)$ & $0.0126(9)$ \\
\hline $\mathrm{C} 21$ & $0.0567(14)$ & 0.0473 (14) & $0.0247(11)$ & $0.0203(11)$ & $0.0155(10)$ & $0.0182(10)$ \\
\hline $\mathrm{C} 22$ & $0.0270(10)$ & $0.0370(11)$ & $0.0193(9)$ & $0.0072(8)$ & 0.0087 (8) & $0.0103(8)$ \\
\hline $\mathrm{C} 23$ & $0.0322(12)$ & $0.0445(13)$ & $0.0332(12)$ & $0.0010(10)$ & $0.0082(10)$ & $0.0157(10)$ \\
\hline $\mathrm{C} 24$ & $0.0351(14)$ & $0.088(2)$ & $0.0525(16)$ & $-0.0016(13)$ & $0.0166(12)$ & $0.0152(14)$ \\
\hline $\mathrm{C} 25$ & $0.0543(16)$ & $0.0477(15)$ & $0.0515(16)$ & $-0.0070(12)$ & 0.0008 (13) & $0.0275(13)$ \\
\hline $\mathrm{C} 26$ & $0.085(2)$ & $0.0339(14)$ & 0.0665 (19) & $0.0105(14)$ & $0.0025(17)$ & $0.0063(13)$ \\
\hline $\mathrm{C} 27$ & $0.089(2)$ & $0.0421(17)$ & $0.090(2)$ & $-0.0142(16)$ & $-0.0010(19)$ & $0.0186(16)$ \\
\hline $\mathrm{C} 28$ & $0.0318(11)$ & 0.0308 (11) & $0.0260(10)$ & $0.0066(9)$ & $0.0114(9)$ & $0.0057(8)$ \\
\hline
\end{tabular}




$\begin{array}{lllllll}\mathrm{C} 29 & 0.0319(12) & 0.0713(17) & 0.0274(11) & 0.0010(11) & 0.0052(10) & 0.0261(11) \\ \mathrm{C} 30 & 0.0395(13) & 0.0511(14) & 0.0331(12) & 0.0005(10) & 0.0166(10) & 0.0145(10)\end{array}$

Geometric parameters $\left(A,{ }^{o}\right)$

\begin{tabular}{|c|c|c|c|}
\hline $\mathrm{C} 11-\mathrm{C} 1$ & $1.7336(19)$ & $\mathrm{C} 11-\mathrm{C} 12$ & $1.490(3)$ \\
\hline $\mathrm{C} 12-\mathrm{C} 16$ & $1.743(2)$ & $\mathrm{C} 11-\mathrm{H} 11 \mathrm{~A}$ & 0.9900 \\
\hline $\mathrm{O} 1-\mathrm{C} 7$ & $1.424(2)$ & C11-H11B & 0.9900 \\
\hline $\mathrm{O} 1-\mathrm{H} 1$ & 0.8400 & $\mathrm{C} 12-\mathrm{H} 12 \mathrm{~A}$ & 0.9900 \\
\hline $\mathrm{O} 2-\mathrm{C} 22$ & $1.427(2)$ & $\mathrm{C} 12-\mathrm{H} 12 \mathrm{~B}$ & 0.9900 \\
\hline $\mathrm{O} 2-\mathrm{H} 2$ & 0.8400 & $\mathrm{C} 13-\mathrm{H} 13 \mathrm{~A}$ & 0.9900 \\
\hline $\mathrm{N} 1-\mathrm{C} 14$ & $1.333(2)$ & $\mathrm{C} 13-\mathrm{H} 13 \mathrm{~B}$ & 0.9900 \\
\hline $\mathrm{N} 1-\mathrm{N} 2$ & $1.364(2)$ & C14-H14 & 0.9500 \\
\hline $\mathrm{N} 1-\mathrm{C} 13$ & $1.454(2)$ & $\mathrm{C} 15-\mathrm{H} 15$ & 0.9500 \\
\hline $\mathrm{N} 2-\mathrm{C} 15$ & $1.313(2)$ & $\mathrm{C} 16-\mathrm{C} 17$ & $1.370(3)$ \\
\hline N3-C14 & $1.312(3)$ & $\mathrm{C} 16-\mathrm{C} 21$ & $1.377(3)$ \\
\hline N3-C15 & $1.360(2)$ & $\mathrm{C} 17-\mathrm{C} 18$ & $1.378(3)$ \\
\hline $\mathrm{N} 4-\mathrm{C} 29$ & $1.322(3)$ & $\mathrm{C} 17-\mathrm{H} 17$ & 0.9500 \\
\hline N4-N5 & $1.351(2)$ & $\mathrm{C} 18-\mathrm{C} 19$ & $1.379(3)$ \\
\hline $\mathrm{N} 4-\mathrm{C} 28$ & $1.465(2)$ & $\mathrm{C} 18-\mathrm{H} 18$ & 0.9500 \\
\hline $\mathrm{N} 5-\mathrm{C} 30$ & $1.309(3)$ & $\mathrm{C} 19-\mathrm{C} 20$ & $1.394(3)$ \\
\hline $\mathrm{N} 6-\mathrm{C} 29$ & $1.326(3)$ & $\mathrm{C} 19-\mathrm{C} 22$ & $1.522(3)$ \\
\hline N6-C30 & $1.328(3)$ & $\mathrm{C} 20-\mathrm{C} 21$ & $1.374(3)$ \\
\hline $\mathrm{C} 1-\mathrm{C} 6$ & $1.382(3)$ & $\mathrm{C} 20-\mathrm{H} 20$ & 0.9500 \\
\hline $\mathrm{C} 1-\mathrm{C} 2$ & $1.383(3)$ & $\mathrm{C} 21-\mathrm{H} 21$ & 0.9500 \\
\hline $\mathrm{C} 2-\mathrm{C} 3$ & $1.380(3)$ & $\mathrm{C} 22-\mathrm{C} 28$ & $1.523(3)$ \\
\hline $\mathrm{C} 2-\mathrm{H} 2 \mathrm{~A}$ & 0.9500 & $\mathrm{C} 22-\mathrm{C} 23$ & $1.563(3)$ \\
\hline $\mathrm{C} 3-\mathrm{C} 4$ & $1.383(3)$ & $\mathrm{C} 23-\mathrm{C} 24$ & $1.505(3)$ \\
\hline $\mathrm{C} 3-\mathrm{H} 3$ & 0.9500 & $\mathrm{C} 23-\mathrm{C} 25$ & $1.509(4)$ \\
\hline $\mathrm{C} 4-\mathrm{C} 5$ & $1.404(2)$ & $\mathrm{C} 23-\mathrm{H} 23$ & 1.0000 \\
\hline $\mathrm{C} 4-\mathrm{C} 7$ & $1.525(2)$ & $\mathrm{C} 24-\mathrm{H} 24 \mathrm{~A}$ & 0.9800 \\
\hline $\mathrm{C} 5-\mathrm{C} 6$ & $1.376(3)$ & $\mathrm{C} 24-\mathrm{H} 24 \mathrm{~B}$ & 0.9800 \\
\hline $\mathrm{C} 5-\mathrm{H} 5$ & 0.9500 & $\mathrm{C} 24-\mathrm{H} 24 \mathrm{C}$ & 0.9800 \\
\hline C6-H6 & 0.9500 & $\mathrm{C} 25-\mathrm{C} 26$ & $1.497(4)$ \\
\hline $\mathrm{C} 7-\mathrm{C} 13$ & $1.546(3)$ & $\mathrm{C} 25-\mathrm{C} 27$ & $1.509(4)$ \\
\hline $\mathrm{C} 7-\mathrm{C} 8$ & $1.553(2)$ & $\mathrm{C} 25-\mathrm{H} 25$ & 1.0000 \\
\hline $\mathrm{C} 8-\mathrm{C} 10$ & $1.511(3)$ & $\mathrm{C} 26-\mathrm{C} 27$ & $1.487(5)$ \\
\hline $\mathrm{C} 8-\mathrm{C} 9$ & $1.526(3)$ & $\mathrm{C} 26-\mathrm{H} 26 \mathrm{~A}$ & 0.9900 \\
\hline $\mathrm{C} 8-\mathrm{H} 8$ & 1.0000 & $\mathrm{C} 26-\mathrm{H} 26 \mathrm{~B}$ & 0.9900 \\
\hline C9-H9A & 0.9800 & $\mathrm{C} 27-\mathrm{H} 27 \mathrm{~A}$ & 0.9900 \\
\hline C9- & 0.9800 & $\mathrm{C} 27-\mathrm{H} 27 \mathrm{~B}$ & 0.9900 \\
\hline C9- $\mathrm{H} 9 \mathrm{C}$ & 0.9800 & $\mathrm{C} 28-\mathrm{H} 28 \mathrm{~A}$ & 0.9900 \\
\hline $\mathrm{C} 10-\mathrm{C} 11$ & $1.493(3)$ & $\mathrm{C} 28-\mathrm{H} 28 \mathrm{~B}$ & 0.9900 \\
\hline $\mathrm{C} 10-\mathrm{C} 12$ & $1.494(3)$ & $\mathrm{C} 29-\mathrm{H} 29$ & 0.9500 \\
\hline $\mathrm{C} 10-\mathrm{H} 10$ & 1.0000 & $\mathrm{C} 30-\mathrm{H} 30$ & 0.9500 \\
\hline $\mathrm{C} 7-\mathrm{O} 1-\mathrm{H} 1$ & 109.5 & $\mathrm{~N} 3-\mathrm{C} 14-\mathrm{N} 1$ & $111.26(17)$ \\
\hline $\mathrm{C} 22-\mathrm{O} 2-\mathrm{H} 2$ & 109.5 & $\mathrm{~N} 3-\mathrm{C} 14-\mathrm{H} 14$ & 124.4 \\
\hline
\end{tabular}




\begin{tabular}{|c|c|}
\hline $\mathrm{C} 14-\mathrm{N} 1-\mathrm{N} 2$ & $109.19(16)$ \\
\hline $\mathrm{C} 14-\mathrm{N} 1-\mathrm{C} 13$ & $128.64(16)$ \\
\hline $\mathrm{N} 2-\mathrm{N} 1-\mathrm{C} 13$ & $121.83(15)$ \\
\hline $\mathrm{C} 15-\mathrm{N} 2-\mathrm{N} 1$ & $102.17(15)$ \\
\hline $\mathrm{C} 14-\mathrm{N} 3-\mathrm{C} 15$ & $102.09(16)$ \\
\hline $\mathrm{C} 29-\mathrm{N} 4-\mathrm{N} 5$ & $108.75(16)$ \\
\hline $\mathrm{C} 29-\mathrm{N} 4-\mathrm{C} 28$ & $130.05(17)$ \\
\hline $\mathrm{N} 5-\mathrm{N} 4-\mathrm{C} 28$ & $121.17(15)$ \\
\hline $\mathrm{C} 30-\mathrm{N} 5-\mathrm{N} 4$ & $102.58(17)$ \\
\hline $\mathrm{C} 29-\mathrm{N} 6-\mathrm{C} 30$ & $101.93(18)$ \\
\hline $\mathrm{C} 6-\mathrm{C} 1-\mathrm{C} 2$ & $120.79(18)$ \\
\hline $\mathrm{C} 6-\mathrm{C} 1-\mathrm{Cl} 1$ & $119.79(14)$ \\
\hline $\mathrm{C} 2-\mathrm{C} 1-\mathrm{Cl} 1$ & $119.41(15)$ \\
\hline $\mathrm{C} 3-\mathrm{C} 2-\mathrm{C} 1$ & $118.83(18)$ \\
\hline $\mathrm{C} 3-\mathrm{C} 2-\mathrm{H} 2 \mathrm{~A}$ & 120.6 \\
\hline $\mathrm{C} 1-\mathrm{C} 2-\mathrm{H} 2 \mathrm{~A}$ & 120.6 \\
\hline $\mathrm{C} 2-\mathrm{C} 3-\mathrm{C} 4$ & $122.27(17)$ \\
\hline $\mathrm{C} 2-\mathrm{C} 3-\mathrm{H} 3$ & 118.9 \\
\hline $\mathrm{C} 4-\mathrm{C} 3-\mathrm{H} 3$ & 118.9 \\
\hline $\mathrm{C} 3-\mathrm{C} 4-\mathrm{C} 5$ & $117.29(17)$ \\
\hline $\mathrm{C} 3-\mathrm{C} 4-\mathrm{C} 7$ & $120.59(16)$ \\
\hline $\mathrm{C} 5-\mathrm{C} 4-\mathrm{C} 7$ & $122.02(16)$ \\
\hline $\mathrm{C} 6-\mathrm{C} 5-\mathrm{C} 4$ & $121.39(17)$ \\
\hline $\mathrm{C} 6-\mathrm{C} 5-\mathrm{H} 5$ & 119.3 \\
\hline $\mathrm{C} 4-\mathrm{C} 5-\mathrm{H} 5$ & 119.3 \\
\hline $\mathrm{C} 5-\mathrm{C} 6-\mathrm{C} 1$ & $119.38(17)$ \\
\hline $\mathrm{C} 5-\mathrm{C} 6-\mathrm{H} 6$ & 120.3 \\
\hline $\mathrm{C} 1-\mathrm{C} 6-\mathrm{H} 6$ & 120.3 \\
\hline $\mathrm{O} 1-\mathrm{C} 7-\mathrm{C} 4$ & $110.59(14)$ \\
\hline $\mathrm{O} 1-\mathrm{C} 7-\mathrm{C} 13$ & $108.50(14)$ \\
\hline $\mathrm{C} 4-\mathrm{C} 7-\mathrm{C} 13$ & $106.44(13)$ \\
\hline $\mathrm{O} 1-\mathrm{C} 7-\mathrm{C} 8$ & $105.33(13)$ \\
\hline $\mathrm{C} 4-\mathrm{C} 7-\mathrm{C} 8$ & $113.17(15)$ \\
\hline $\mathrm{C} 13-\mathrm{C} 7-\mathrm{C} 8$ & $112.78(15)$ \\
\hline $\mathrm{C} 10-\mathrm{C} 8-\mathrm{C} 9$ & $110.00(16)$ \\
\hline $\mathrm{C} 10-\mathrm{C} 8-\mathrm{C} 7$ & $113.93(14)$ \\
\hline $\mathrm{C} 9-\mathrm{C} 8-\mathrm{C} 7$ & $111.32(15)$ \\
\hline $\mathrm{C} 10-\mathrm{C} 8-\mathrm{H} 8$ & 107.1 \\
\hline $\mathrm{C} 9-\mathrm{C} 8-\mathrm{H} 8$ & 107.1 \\
\hline $\mathrm{C} 7-\mathrm{C} 8-\mathrm{H} 8$ & 107.1 \\
\hline $\mathrm{C} 8-\mathrm{C} 9-\mathrm{H} 9 \mathrm{~A}$ & 109.5 \\
\hline $\mathrm{C} 8-\mathrm{C} 9-\mathrm{H} 9 \mathrm{~B}$ & 109.5 \\
\hline $\mathrm{H} 9 \mathrm{~A}-\mathrm{C} 9-\mathrm{H} 9 \mathrm{~B}$ & 109.5 \\
\hline $\mathrm{C} 8-\mathrm{C} 9-\mathrm{H} 9 \mathrm{C}$ & 109.5 \\
\hline $\mathrm{H} 9 \mathrm{~A}-\mathrm{C} 9-\mathrm{H} 9 \mathrm{C}$ & 109.5 \\
\hline $\mathrm{H} 9 \mathrm{~B}-\mathrm{C} 9-\mathrm{H} 9 \mathrm{C}$ & 109.5 \\
\hline $\mathrm{C} 11-\mathrm{C} 10-\mathrm{C} 12$ & $59.86(15)$ \\
\hline $\mathrm{C} 11-\mathrm{C} 10-\mathrm{C} 8$ & $121.87(19)$ \\
\hline
\end{tabular}

\begin{tabular}{|c|c|}
\hline $\mathrm{N} 1-\mathrm{C} 14-\mathrm{H} 14$ & 124.4 \\
\hline $\mathrm{N} 2-\mathrm{C} 15-\mathrm{N} 3$ & $115.29(17)$ \\
\hline $\mathrm{N} 2-\mathrm{C} 15-\mathrm{H} 15$ & 122.4 \\
\hline $\mathrm{N} 3-\mathrm{C} 15-\mathrm{H} 15$ & 122.4 \\
\hline $\mathrm{C} 17-\mathrm{C} 16-\mathrm{C} 21$ & $120.6(2)$ \\
\hline $\mathrm{C} 17-\mathrm{C} 16-\mathrm{Cl} 2$ & $120.43(17)$ \\
\hline $\mathrm{C} 21-\mathrm{C} 16-\mathrm{Cl} 2$ & $118.95(17)$ \\
\hline $\mathrm{C} 16-\mathrm{C} 17-\mathrm{C} 18$ & $119.6(2)$ \\
\hline $\mathrm{C} 16-\mathrm{C} 17-\mathrm{H} 17$ & 120.2 \\
\hline $\mathrm{C} 18-\mathrm{C} 17-\mathrm{H} 17$ & 120.2 \\
\hline $\mathrm{C} 17-\mathrm{C} 18-\mathrm{C} 19$ & $121.46(19)$ \\
\hline $\mathrm{C} 17-\mathrm{C} 18-\mathrm{H} 18$ & 119.3 \\
\hline $\mathrm{C} 19-\mathrm{C} 18-\mathrm{H} 18$ & 119.3 \\
\hline $\mathrm{C} 18-\mathrm{C} 19-\mathrm{C} 20$ & $117.53(19)$ \\
\hline $\mathrm{C} 18-\mathrm{C} 19-\mathrm{C} 22$ & $122.35(17)$ \\
\hline $\mathrm{C} 20-\mathrm{C} 19-\mathrm{C} 22$ & $120.12(17)$ \\
\hline $\mathrm{C} 21-\mathrm{C} 20-\mathrm{C} 19$ & $121.63(19)$ \\
\hline $\mathrm{C} 21-\mathrm{C} 20-\mathrm{H} 20$ & 119.2 \\
\hline $\mathrm{C} 19-\mathrm{C} 20-\mathrm{H} 20$ & 119.2 \\
\hline $\mathrm{C} 20-\mathrm{C} 21-\mathrm{C} 16$ & $119.11(19)$ \\
\hline $\mathrm{C} 20-\mathrm{C} 21-\mathrm{H} 21$ & 120.4 \\
\hline $\mathrm{C} 16-\mathrm{C} 21-\mathrm{H} 21$ & 120.4 \\
\hline $\mathrm{O} 2-\mathrm{C} 22-\mathrm{C} 19$ & $112.11(15)$ \\
\hline $\mathrm{O} 2-\mathrm{C} 22-\mathrm{C} 28$ & $104.26(16)$ \\
\hline $\mathrm{C} 19-\mathrm{C} 22-\mathrm{C} 28$ & $110.58(16)$ \\
\hline $\mathrm{O} 2-\mathrm{C} 22-\mathrm{C} 23$ & $111.03(16)$ \\
\hline $\mathrm{C} 19-\mathrm{C} 22-\mathrm{C} 23$ & $109.95(16)$ \\
\hline $\mathrm{C} 28-\mathrm{C} 22-\mathrm{C} 23$ & $108.73(16)$ \\
\hline $\mathrm{C} 24-\mathrm{C} 23-\mathrm{C} 25$ & $111.2(2)$ \\
\hline $\mathrm{C} 24-\mathrm{C} 23-\mathrm{C} 22$ & $111.75(19)$ \\
\hline $\mathrm{C} 25-\mathrm{C} 23-\mathrm{C} 22$ & $112.09(19)$ \\
\hline $\mathrm{C} 24-\mathrm{C} 23-\mathrm{H} 23$ & 107.2 \\
\hline $\mathrm{C} 25-\mathrm{C} 23-\mathrm{H} 23$ & 107.2 \\
\hline $\mathrm{C} 22-\mathrm{C} 23-\mathrm{H} 23$ & 107.2 \\
\hline $\mathrm{C} 23-\mathrm{C} 24-\mathrm{H} 24 \mathrm{~A}$ & 109.5 \\
\hline $\mathrm{C} 23-\mathrm{C} 24-\mathrm{H} 24 \mathrm{~B}$ & 109.5 \\
\hline $\mathrm{H} 24 \mathrm{~A}-\mathrm{C} 24-\mathrm{H} 24 \mathrm{~B}$ & 109.5 \\
\hline $\mathrm{C} 23-\mathrm{C} 24-\mathrm{H} 24 \mathrm{C}$ & 109.5 \\
\hline $\mathrm{H} 24 \mathrm{~A}-\mathrm{C} 24-\mathrm{H} 24 \mathrm{C}$ & 109.5 \\
\hline $\mathrm{H} 24 \mathrm{~B}-\mathrm{C} 24-\mathrm{H} 24 \mathrm{C}$ & 109.5 \\
\hline $\mathrm{C} 26-\mathrm{C} 25-\mathrm{C} 27$ & $59.3(2)$ \\
\hline $\mathrm{C} 26-\mathrm{C} 25-\mathrm{C} 23$ & $121.6(2)$ \\
\hline $\mathrm{C} 27-\mathrm{C} 25-\mathrm{C} 23$ & $118.7(2)$ \\
\hline $\mathrm{C} 26-\mathrm{C} 25-\mathrm{H} 25$ & 115.2 \\
\hline $\mathrm{C} 27-\mathrm{C} 25-\mathrm{H} 25$ & 115.2 \\
\hline $\mathrm{C} 23-\mathrm{C} 25-\mathrm{H} 25$ & 115.2 \\
\hline $\mathrm{C} 27-\mathrm{C} 26-\mathrm{C} 25$ & $60.7(2)$ \\
\hline $\mathrm{C} 27-\mathrm{C} 26-\mathrm{H} 26 \mathrm{~A}$ & 117.7 \\
\hline
\end{tabular}




\begin{tabular}{|c|c|c|c|}
\hline $\mathrm{C} 12-\mathrm{C} 10-\mathrm{C} 8$ & $118.46(17)$ & $\mathrm{C} 25-\mathrm{C} 26-\mathrm{H} 26 \mathrm{~A}$ & 117.7 \\
\hline $\mathrm{C} 11-\mathrm{C} 10-\mathrm{H} 10$ & 115.1 & $\mathrm{C} 27-\mathrm{C} 26-\mathrm{H} 26 \mathrm{~B}$ & 117.7 \\
\hline $\mathrm{C} 12-\mathrm{C} 10-\mathrm{H} 10$ & 115.1 & $\mathrm{C} 25-\mathrm{C} 26-\mathrm{H} 26 \mathrm{~B}$ & 117.7 \\
\hline $\mathrm{C} 8-\mathrm{C} 10-\mathrm{H} 10$ & 115.1 & $\mathrm{H} 26 \mathrm{~A}-\mathrm{C} 26-\mathrm{H} 26 \mathrm{~B}$ & 114.8 \\
\hline $\mathrm{C} 12-\mathrm{C} 11-\mathrm{C} 10$ & $60.11(14)$ & $\mathrm{C} 26-\mathrm{C} 27-\mathrm{C} 25$ & $59.95(19)$ \\
\hline $\mathrm{C} 12-\mathrm{C} 11-\mathrm{H} 11 \mathrm{~A}$ & 117.8 & $\mathrm{C} 26-\mathrm{C} 27-\mathrm{H} 27 \mathrm{~A}$ & 117.8 \\
\hline $\mathrm{C} 10-\mathrm{C} 11-\mathrm{H} 11 \mathrm{~A}$ & 117.8 & $\mathrm{C} 25-\mathrm{C} 27-\mathrm{H} 27 \mathrm{~A}$ & 117.8 \\
\hline $\mathrm{C} 12-\mathrm{C} 11-\mathrm{H} 11 \mathrm{~B}$ & 117.8 & $\mathrm{C} 26-\mathrm{C} 27-\mathrm{H} 27 \mathrm{~B}$ & 117.8 \\
\hline $\mathrm{C} 10-\mathrm{C} 11-\mathrm{H} 11 \mathrm{~B}$ & 117.8 & $\mathrm{C} 25-\mathrm{C} 27-\mathrm{H} 27 \mathrm{~B}$ & 117.8 \\
\hline $\mathrm{H} 11 \mathrm{~A}-\mathrm{C} 11-\mathrm{H} 11 \mathrm{~B}$ & 114.9 & $\mathrm{H} 27 \mathrm{~A}-\mathrm{C} 27-\mathrm{H} 27 \mathrm{~B}$ & 114.9 \\
\hline $\mathrm{C} 11-\mathrm{C} 12-\mathrm{C} 10$ & $60.03(14)$ & $\mathrm{N} 4-\mathrm{C} 28-\mathrm{C} 22$ & $113.94(15)$ \\
\hline $\mathrm{C} 11-\mathrm{C} 12-\mathrm{H} 12 \mathrm{~A}$ & 117.8 & $\mathrm{~N} 4-\mathrm{C} 28-\mathrm{H} 28 \mathrm{~A}$ & 108.8 \\
\hline $\mathrm{C} 10-\mathrm{C} 12-\mathrm{H} 12 \mathrm{~A}$ & 117.8 & $\mathrm{C} 22-\mathrm{C} 28-\mathrm{H} 28 \mathrm{~A}$ & 108.8 \\
\hline $\mathrm{C} 11-\mathrm{C} 12-\mathrm{H} 12 \mathrm{~B}$ & 117.8 & $\mathrm{~N} 4-\mathrm{C} 28-\mathrm{H} 28 \mathrm{~B}$ & 108.8 \\
\hline $\mathrm{C} 10-\mathrm{C} 12-\mathrm{H} 12 \mathrm{~B}$ & 117.8 & $\mathrm{C} 22-\mathrm{C} 28-\mathrm{H} 28 \mathrm{~B}$ & 108.8 \\
\hline $\mathrm{H} 12 \mathrm{~A}-\mathrm{C} 12-\mathrm{H} 12 \mathrm{~B}$ & 114.9 & $\mathrm{H} 28 \mathrm{~A}-\mathrm{C} 28-\mathrm{H} 28 \mathrm{~B}$ & 107.7 \\
\hline $\mathrm{N} 1-\mathrm{C} 13-\mathrm{C} 7$ & $114.60(14)$ & $\mathrm{N} 4-\mathrm{C} 29-\mathrm{N} 6$ & $111.02(19)$ \\
\hline $\mathrm{N} 1-\mathrm{C} 13-\mathrm{H} 13 \mathrm{~A}$ & 108.6 & $\mathrm{~N} 4-\mathrm{C} 29-\mathrm{H} 29$ & 124.5 \\
\hline $\mathrm{C} 7-\mathrm{C} 13-\mathrm{H} 13 \mathrm{~A}$ & 108.6 & $\mathrm{~N} 6-\mathrm{C} 29-\mathrm{H} 29$ & 124.5 \\
\hline $\mathrm{N} 1-\mathrm{C} 13-\mathrm{H} 13 \mathrm{~B}$ & 108.6 & $\mathrm{~N} 5-\mathrm{C} 30-\mathrm{N} 6$ & $115.70(19)$ \\
\hline C7-C13-H13B & 108.6 & $\mathrm{~N} 5-\mathrm{C} 30-\mathrm{H} 30$ & 122.2 \\
\hline $\mathrm{H} 13 \mathrm{~A}-\mathrm{C} 13-\mathrm{H} 13 \mathrm{~B}$ & 107.6 & $\mathrm{~N} 6-\mathrm{C} 30-\mathrm{H} 30$ & 122.2 \\
\hline $\mathrm{C} 14-\mathrm{N} 1-\mathrm{N} 2-\mathrm{C} 15$ & $0.54(18)$ & $\mathrm{N} 1-\mathrm{N} 2-\mathrm{C} 15-\mathrm{N} 3$ & $-0.4(2)$ \\
\hline $\mathrm{C} 13-\mathrm{N} 1-\mathrm{N} 2-\mathrm{C} 15$ & $174.41(15)$ & $\mathrm{C} 14-\mathrm{N} 3-\mathrm{C} 15-\mathrm{N} 2$ & $0.2(2)$ \\
\hline $\mathrm{C} 29-\mathrm{N} 4-\mathrm{N} 5-\mathrm{C} 30$ & $1.2(3)$ & $\mathrm{C} 21-\mathrm{C} 16-\mathrm{C} 17-\mathrm{C} 18$ & $0.4(4)$ \\
\hline $\mathrm{C} 28-\mathrm{N} 4-\mathrm{N} 5-\mathrm{C} 30$ & $-177.3(2)$ & $\mathrm{Cl} 2-\mathrm{C} 16-\mathrm{C} 17-\mathrm{C} 18$ & $-179.79(18)$ \\
\hline $\mathrm{C} 6-\mathrm{C} 1-\mathrm{C} 2-\mathrm{C} 3$ & $1.9(3)$ & $\mathrm{C} 16-\mathrm{C} 17-\mathrm{C} 18-\mathrm{C} 19$ & $-2.0(4)$ \\
\hline $\mathrm{C} 11-\mathrm{C} 1-\mathrm{C} 2-\mathrm{C} 3$ & $-177.79(15)$ & $\mathrm{C} 17-\mathrm{C} 18-\mathrm{C} 19-\mathrm{C} 20$ & $2.3(3)$ \\
\hline $\mathrm{C} 1-\mathrm{C} 2-\mathrm{C} 3-\mathrm{C} 4$ & $0.0(3)$ & $\mathrm{C} 17-\mathrm{C} 18-\mathrm{C} 19-\mathrm{C} 22$ & $-176.9(2)$ \\
\hline $\mathrm{C} 2-\mathrm{C} 3-\mathrm{C} 4-\mathrm{C} 5$ & $-1.7(3)$ & $\mathrm{C} 18-\mathrm{C} 19-\mathrm{C} 20-\mathrm{C} 21$ & $-1.0(3)$ \\
\hline $\mathrm{C} 2-\mathrm{C} 3-\mathrm{C} 4-\mathrm{C} 7$ & $174.90(17)$ & $\mathrm{C} 22-\mathrm{C} 19-\mathrm{C} 20-\mathrm{C} 21$ & $178.2(2)$ \\
\hline $\mathrm{C} 3-\mathrm{C} 4-\mathrm{C} 5-\mathrm{C} 6$ & $1.6(3)$ & $\mathrm{C} 19-\mathrm{C} 20-\mathrm{C} 21-\mathrm{C} 16$ & $-0.5(4)$ \\
\hline $\mathrm{C} 7-\mathrm{C} 4-\mathrm{C} 5-\mathrm{C} 6$ & $-174.93(17)$ & $\mathrm{C} 17-\mathrm{C} 16-\mathrm{C} 21-\mathrm{C} 20$ & $0.8(4)$ \\
\hline $\mathrm{C} 4-\mathrm{C} 5-\mathrm{C} 6-\mathrm{C} 1$ & $0.2(3)$ & $\mathrm{C} 12-\mathrm{C} 16-\mathrm{C} 21-\mathrm{C} 20$ & $-179.00(18)$ \\
\hline $\mathrm{C} 2-\mathrm{C} 1-\mathrm{C} 6-\mathrm{C} 5$ & $-2.0(3)$ & $\mathrm{C} 18-\mathrm{C} 19-\mathrm{C} 22-\mathrm{O} 2$ & $-9.3(3)$ \\
\hline $\mathrm{C} 11-\mathrm{C} 1-\mathrm{C} 6-\mathrm{C} 5$ & $177.71(14)$ & $\mathrm{C} 20-\mathrm{C} 19-\mathrm{C} 22-\mathrm{O} 2$ & $171.49(17)$ \\
\hline $\mathrm{C} 3-\mathrm{C} 4-\mathrm{C} 7-\mathrm{O} 1$ & $-17.5(2)$ & $\mathrm{C} 18-\mathrm{C} 19-\mathrm{C} 22-\mathrm{C} 28$ & $-125.2(2)$ \\
\hline $\mathrm{C} 5-\mathrm{C} 4-\mathrm{C} 7-\mathrm{O} 1$ & $158.99(16)$ & $\mathrm{C} 20-\mathrm{C} 19-\mathrm{C} 22-\mathrm{C} 28$ & $55.6(2)$ \\
\hline $\mathrm{C} 3-\mathrm{C} 4-\mathrm{C} 7-\mathrm{C} 13$ & $-135.12(17)$ & $\mathrm{C} 18-\mathrm{C} 19-\mathrm{C} 22-\mathrm{C} 23$ & $114.7(2)$ \\
\hline $\mathrm{C} 5-\mathrm{C} 4-\mathrm{C} 7-\mathrm{C} 13$ & $41.3(2)$ & $\mathrm{C} 20-\mathrm{C} 19-\mathrm{C} 22-\mathrm{C} 23$ & $-64.5(2)$ \\
\hline $\mathrm{C} 3-\mathrm{C} 4-\mathrm{C} 7-\mathrm{C} 8$ & $100.45(19)$ & $\mathrm{O} 2-\mathrm{C} 22-\mathrm{C} 23-\mathrm{C} 24$ & $65.7(2)$ \\
\hline $\mathrm{C} 5-\mathrm{C} 4-\mathrm{C} 7-\mathrm{C} 8$ & $-83.1(2)$ & $\mathrm{C} 19-\mathrm{C} 22-\mathrm{C} 23-\mathrm{C} 24$ & $-59.0(2)$ \\
\hline $\mathrm{O} 1-\mathrm{C} 7-\mathrm{C} 8-\mathrm{C} 10$ & $-179.60(15)$ & $\mathrm{C} 28-\mathrm{C} 22-\mathrm{C} 23-\mathrm{C} 24$ & $179.8(2)$ \\
\hline $\mathrm{C} 4-\mathrm{C} 7-\mathrm{C} 8-\mathrm{C} 10$ & $59.5(2)$ & $\mathrm{O} 2-\mathrm{C} 22-\mathrm{C} 23-\mathrm{C} 25$ & $-59.9(2)$ \\
\hline $\mathrm{C} 13-\mathrm{C} 7-\mathrm{C} 8-\mathrm{C} 10$ & $-61.4(2)$ & $\mathrm{C} 19-\mathrm{C} 22-\mathrm{C} 23-\mathrm{C} 25$ & $175.41(19)$ \\
\hline $\mathrm{O} 1-\mathrm{C} 7-\mathrm{C} 8-\mathrm{C} 9$ & $55.3(2)$ & $\mathrm{C} 28-\mathrm{C} 22-\mathrm{C} 23-\mathrm{C} 25$ & $54.2(2)$ \\
\hline $\mathrm{C} 4-\mathrm{C} 7-\mathrm{C} 8-\mathrm{C} 9$ & $-65.6(2)$ & $\mathrm{C} 24-\mathrm{C} 23-\mathrm{C} 25-\mathrm{C} 26$ & $142.2(2)$ \\
\hline
\end{tabular}




$$
\begin{aligned}
& \mathrm{C} 13-\mathrm{C} 7-\mathrm{C} 8-\mathrm{C} 9 \\
& \mathrm{C} 9-\mathrm{C} 8-\mathrm{C} 10-\mathrm{C} 11 \\
& \mathrm{C} 7-\mathrm{C} 8-\mathrm{C} 10-\mathrm{C} 11 \\
& \mathrm{C} 9-\mathrm{C} 8-\mathrm{C} 10-\mathrm{C} 12 \\
& \mathrm{C} 7-\mathrm{C} 8-\mathrm{C} 10-\mathrm{C} 12 \\
& \mathrm{C} 8-\mathrm{C} 10-\mathrm{C} 11-\mathrm{C} 12 \\
& \mathrm{C} 8-\mathrm{C} 10-\mathrm{C} 12-\mathrm{C} 11 \\
& \mathrm{C} 14-\mathrm{N} 1-\mathrm{C} 13-\mathrm{C} 7 \\
& \mathrm{~N} 2-\mathrm{N} 1-\mathrm{C} 13-\mathrm{C} 7 \\
& \mathrm{O} 1-\mathrm{C} 7-\mathrm{C} 13-\mathrm{N} 1 \\
& \mathrm{C} 4-\mathrm{C} 7-\mathrm{C} 13-\mathrm{N} 1 \\
& \mathrm{C} 8-\mathrm{C} 7-\mathrm{C} 13-\mathrm{N} 1 \\
& \mathrm{C} 15-\mathrm{N} 3-\mathrm{C} 14-\mathrm{N} 1 \\
& \mathrm{~N} 2-\mathrm{N} 1-\mathrm{C} 14-\mathrm{N} 3 \\
& \mathrm{C} 13-\mathrm{N} 1-\mathrm{C} 14-\mathrm{N} 3
\end{aligned}
$$

$$
\begin{aligned}
& 173.47(16) \\
& -146.64(19) \\
& 87.6(2) \\
& -76.2(2) \\
& 157.97(18) \\
& 106.7(2) \\
& -112.3(2) \\
& -82.3(2) \\
& 105.16(18) \\
& 45.42(19) \\
& 164.46(14) \\
& -70.87(19) \\
& 0.2(2) \\
& -0.5(2) \\
& -173.81(16)
\end{aligned}
$$

$\mathrm{C} 22-\mathrm{C} 23-\mathrm{C} 25-\mathrm{C} 26$
$\mathrm{C} 24-\mathrm{C} 23-\mathrm{C} 25-\mathrm{C} 27$
$\mathrm{C} 22-\mathrm{C} 23-\mathrm{C} 25-\mathrm{C} 27$
$\mathrm{C} 23-\mathrm{C} 25-\mathrm{C} 26-\mathrm{C} 27$
$\mathrm{C} 23-\mathrm{C} 25-\mathrm{C} 27-\mathrm{C} 26$
$\mathrm{C} 29-\mathrm{N} 4-\mathrm{C} 28-\mathrm{C} 22$
$\mathrm{~N} 5-\mathrm{N} 4-\mathrm{C} 28-\mathrm{C} 22$
$\mathrm{O} 2-\mathrm{C} 22-\mathrm{C} 28-\mathrm{N} 4$
$\mathrm{C} 19-\mathrm{C} 22-\mathrm{C} 28-\mathrm{N} 4$
$\mathrm{C} 23-\mathrm{C} 22-\mathrm{C} 28-\mathrm{N} 4$
$\mathrm{~N} 5-\mathrm{N} 4-\mathrm{C} 29-\mathrm{N} 6$
$\mathrm{C} 28-\mathrm{N} 4-\mathrm{C} 29-\mathrm{N} 6$
$\mathrm{C} 30-\mathrm{N} 6-\mathrm{C} 29-\mathrm{N} 4$
$\mathrm{~N} 4-\mathrm{N} 5-\mathrm{C} 30-\mathrm{N} 6$
$\mathrm{C} 29-\mathrm{N} 6-\mathrm{C} 30-\mathrm{N} 5$

$-91.9(3)$

$72.4(3)$

$-161.6(3)$

$-106.9(3)$

$111.7(3)$

$57.1(3)$

$-124.8(2)$

$-71.1(2)$

$49.6(2)$

$170.39(16)$

$-1.7(3)$

$176.6(2)$

$1.4(3)$

$-0.4(3)$

$-0.6(3)$

Hydrogen-bond geometry $\left(A,{ }^{\circ}\right)$

$C g 1$ is the centroid of the $\mathrm{N} 1 / \mathrm{N} 2 / \mathrm{C} 15 / \mathrm{N} 3 / \mathrm{C} 14$ ring.

\begin{tabular}{lllll}
\hline$D-\mathrm{H} \cdots A$ & $D-\mathrm{H}$ & $\mathrm{H} \cdots A$ & $D \cdots A$ & $D-\mathrm{H} \cdots A$ \\
\hline $\mathrm{O} 1-\mathrm{H} 1 \cdots \mathrm{N} 6^{\mathrm{i}}$ & 0.84 & 2.05 & $2.884(2)$ & 171 \\
$\mathrm{O} 2-\mathrm{H} 2 \cdots \mathrm{N} 3^{\mathrm{ii}}$ & 0.84 & 2.07 & $2.856(2)$ & 156 \\
$\mathrm{C} 8-\mathrm{H} 8 \cdots \mathrm{Cl}^{\mathrm{iii}}$ & 1.00 & 2.83 & $3.633(2)$ & 137 \\
$\mathrm{C} 15-\mathrm{H} 15 \cdots \mathrm{O} 2^{\mathrm{iv}}$ & 0.95 & 2.53 & $3.284(2)$ & 137 \\
$\mathrm{C} 13-\mathrm{H} 13 B \cdots C g 1^{\mathrm{v}}$ & 1.00 & 2.91 & $3.473(2)$ & 117 \\
\hline
\end{tabular}

Symmetry codes: (i) $x-1, y, z-1$; (ii) $x, y, z+1$; (iii) $x+1, y, z$; (iv) $-x+2,-y+1,-z+1$; (v) $-x+1,-y+1,-z$. 